\title{
Experimental noise filtering by quantum control
}

\author{
A. Soare ${ }^{1,2 \dagger}$, H. Ball ${ }^{1,2}$, D. Hayes ${ }^{1,2}$, J. Sastrawan ${ }^{1,2}$, M. C. Jarratt ${ }^{1,2}$, J. J. McLoughlin ${ }^{1,2}$, X. Zhen ${ }^{3}$, \\ T. J. Green ${ }^{1,2}$ and M. J. Biercuk ${ }^{1,2 \star}$
}

Extrinsic interference is routinely faced in systems engineering, and a common solution is to rely on a broad class of filtering techniques to afford stability to intrinsically unstable systems or isolate particular signals from a noisy background. Experimentalists leading the development of a new generation of quantum-enabled technologies similarly encounter time-varying noise in realistic laboratory settings. They face substantial challenges in either suppressing such noise for high-fidelity quantum operations ${ }^{1}$ or controllably exploiting it in quantum-enhanced sensing ${ }^{2-4}$ or system identification tasks ${ }^{5,6}$, due to a lack of efficient, validated approaches to understanding and predicting quantum dynamics in the presence of realistic time-varying noise. In this work we use the theory of quantum control engineering 7,8 and experiments with trapped ${ }^{171} \mathrm{Yb}^{+}$ions to study the dynamics of controlled quantum systems. Our results provide the first experimental validation of generalized filter-transfer functions casting arbitrary quantum control operations on qubits as noise spectral filters ${ }^{9,10}$. We demonstrate the utility of these constructs for directly predicting the evolution of a quantum state in a realistic noisy environment as well as for developing novel robust control and sensing protocols. These experiments provide a significant advance in our understanding of the physics underlying controlled quantum dynamics, and unlock new capabilities for the emerging field of quantum systems engineering.

Time-varying noise coupled to quantum systems-typically qubits-generically results in decoherence, or a loss of 'quantumness' of the system. Broadly, one may think of the state of the quantum system becoming randomized through uncontrolled (and often uncontrollable) interactions with the environment during both idle periods and active control operations (Fig. 1a). Despite the ubiquity of this phenomenon, it is a challenging problem to predict the average evolution of a qubit state undergoing a specific, but arbitrary operation in the presence of realistic time-dependent noise-how much randomization does one expect and how well can one perform the target operation? Making such predictions accurately is precisely the capability that experimentalists require in realistic laboratory settings. Moreover, this capability is fundamental to the development of novel control techniques designed to modify or suppress decoherence as researchers attempt to build quantum-enabled technologies for applications such as quantum information and quantum sensing.

These considerations motivate the development of novel engineering-inspired analytic tools enabling a user to accurately predict the behaviour of a controlled quantum system in realistic laboratory environments. Recent work has demonstrated that the average dynamics of a controlled qubit state evolution may be captured using filter-transfer functions (FFs) characterizing the control. The fidelity of an arbitrary operation over duration $\tau$, $\mathcal{F}_{\chi}(\tau) \propto \mathrm{e}^{-\int_{0}^{\infty} \mathrm{d} \omega S(\omega) F(\omega)}$, is degraded owing to frequency-domain spectral overlap between noise in the environment given by a power spectrum $S(\omega)$, and the filter-transfer functions denoted $F(\omega)$ (Methods) $)^{11-14}$.

The FF description of ensemble-average quantum dynamics tremendously simplifies the task of analysing the expected performance of a control protocol in a noisy environment as it permits consideration of control as noise spectral filtering. The FFs themselves may be described using familiar concepts such as frequency passbands, stopbands and filter order, enabling a simple graphical representation of otherwise complex concepts in the dynamics of controlled quantum systems (Fig. 1b). Noise filtering, in practice, is achieved through construction of a control protocol (Fig. 1a) which modifies the controllability of the quantum system by the noisy environment over a defined frequency band. Adjusting $F(\omega)$ and changing its overlap with the noise spectrum thus allows a user to change the average dynamics of the system in a predictable way.

To see the importance of this capability we may consider the various tasks that might be of interest in experimental quantum engineering and the role of noise spectral filtering in these applications. In quantum information an experimentalist may aim to suppress broadband low-frequency noise to maximize the fidelity of a bounded-strength quantum logic operation (Fig. 1b, upper trace), and then calculate the residual error. Alternatively, in quantum-enabled sensing or system identification he or she may perform narrowband spectral characterization of a given operation (Fig. 1b, lower trace), where any change in the measured fidelity under filter application represents the signal of interest $t^{4,6}$.

The intuitive nature of this framework is belied by the challenge of calculating FFs for arbitrary control protocols, generally involving time-domain modulation of control parameters such as the frequency and amplitude of a driving field. The nature of quantum dynamics means that the implemented control framework is generally nonlinear; for instance, one finds complex dynamics in circumstances where the noise and control operations do not commute, such as a driven operation $\left(\propto \sigma_{x}\right)$ in the presence of dephasing noise $\left(\propto \sigma_{z}\right)$. Recent theoretical effort has allowed calculation of FFs for arbitrary single-qubit control and arbitrary universal classical noise $\mathrm{e}^{9,10}$, expanding significantly beyond previous demonstrations restricted to the identity operator in pure-dephasing environments ${ }^{15}$. It

${ }^{1}$ ARC Centre for Engineered Quantum Systems, School of Physics, The University of Sydney, NSW 2006, Australia, ${ }^{2}$ National Measurement Institute, West Lindfield, NSW 2070, Australia, ${ }^{3}$ State Key Laboratory of Low-dimensional Quantum Physics and Department of Physics, Tsinghua University, Beijing 100084, China. These authors contributed equally to this work. Fresent address: Lockheed Martin Corporation, USA.

*e-mail: michael.biercuk@sydney.edu.au 


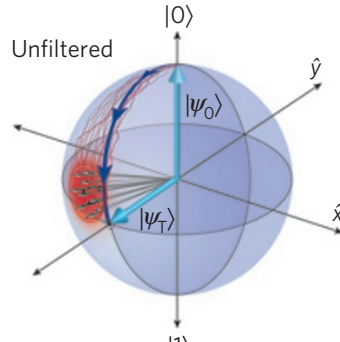

$|1\rangle$

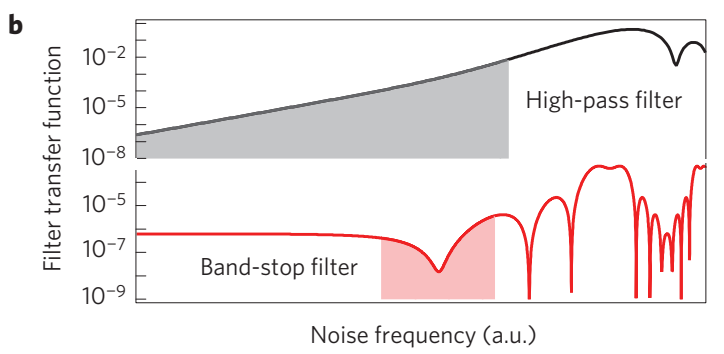

$|0\rangle$

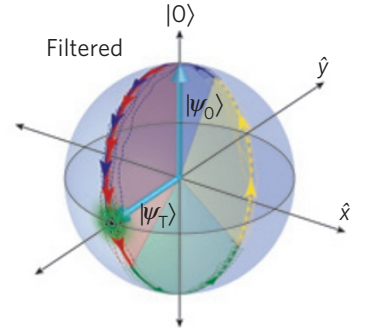

$|1\rangle$

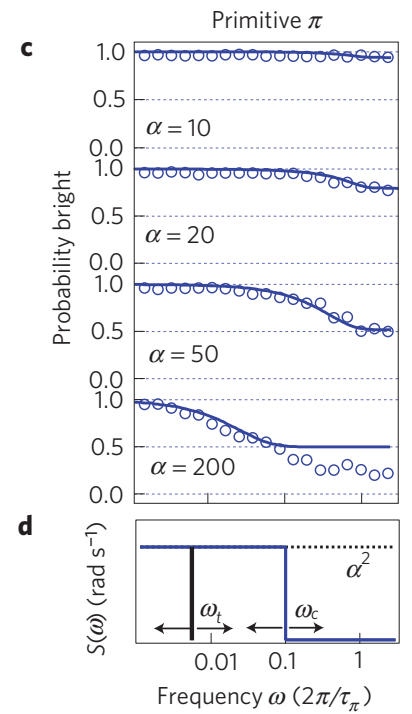

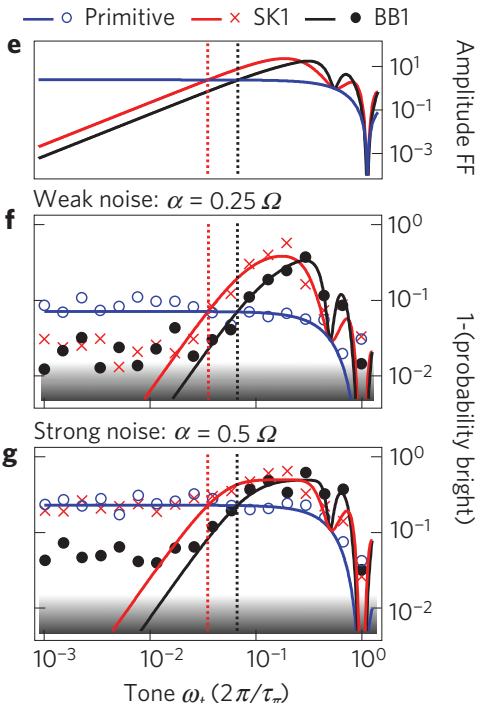

Figure 1 | Noise filters and experimental validation of the predictive power of the filter-transfer function. a, Time-varying noise during an operation

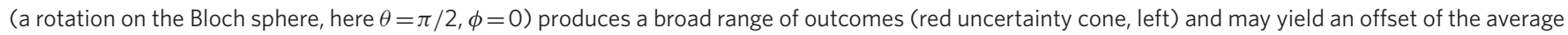

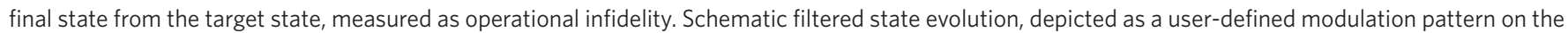
control (coloured segments), changes the measured fidelity by reducing the uncertainty due to noise in a specified band. $\mathbf{b}$, Schematic representation of noise filters of interest-shaded areas represent filter stopbands-crafted by control modulation as indicated above. a.u., arbitrary units. c, Measurements of operational fidelity with engineered dephasing noise for primitive $\pi$ rotation, $|0\rangle \rightarrow|1\rangle$, as a function of dimensionless noise cutoff frequency overlaid with FF-based calculations of $\mathcal{F}_{\chi}(\tau)$. Decay to value 0.5 corresponds to full decoherence. Each data point is the result of averaging over 50 different noise realizations. d, Schematic representation of the quasi-white noise power spectrum with cutoff $\omega_{c}$ employed in c and single-tone power spectrum with

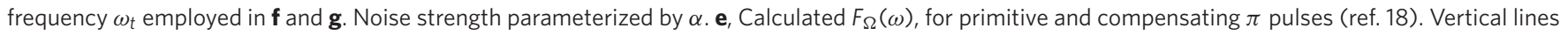
indicate frequencies where $F_{\Omega}(\omega)$ for SK1 (red) and BB1 (black) cross primitive (blue), indicating an expected inversion of performance. f,g, Swept-tone multiplicative amplitude-noise measurements, $S_{\Omega}(\omega) \propto \delta\left(\omega_{t}-\omega\right)$, for various $\pi$ rotations (averaged over 20 noise realizations). Vertical axis is a proxy for measured operational infidelity. Solid lines indicate $1-\mathcal{F}_{\chi}(\tau)$. f, Good agreement is revealed in the weak-noise limit across three decades of frequency, down to the measurement fidelity limit, $\sim 98.5 \%$, indicated by grey shading. Measured gate-error crossover points correspond well with crossovers in the FFs for these gates (vertical dashed lines). Detailed performance differences between protocols in the low-error limit can be revealed through randomized benchmarking, as performed later (Fig. 3). g, In the strong-error limit, first-order approximations are violated and contributions from higher-order Magnus terms contribute to the measured error in the low-frequency limit, yielding (expected) differences between SK1 and BB1 due to Magnus order cancellation and not captured by the FF.

is this more general case where the impact of noise filtering and the FFs may have the most significant impact on the quantum engineering community, and where experimental tests are vital.

In our experimental system, based on the $12.6 \mathrm{GHz}$ qubit transition in ${ }^{171} \mathrm{Yb}^{+}$(Supplementary Methods), we are able to perform quantitative tests of operational fidelity for arbitrary control operations; these may then be compared against calculations of $\mathcal{F}_{\chi}(\tau)$ as a fundamental test of FF validity. A key tool in our studies is bath engineering ${ }^{16}$, in which we add noise with userdefined spectral characteristics to the control system, producing well-controlled unitary dephasing or depolarization.

As a first example (Fig. 1c), experimental measurements of operational fidelity for a $\pi_{x}$-pulse driving qubit population from the dark state to the bright state, $|0\rangle \rightarrow|1\rangle$, in the presence of engineered time-dependent dephasing noise give good agreement with analytic calculation of $\mathcal{F}_{\chi}(\tau)$ using the noise power spectrum and analytic FFs (ref. 10) with no free parameters (Methods). This approach therefore immediately demonstrates the predictive power of the FF formalism.

The FFs for much more complex control, such as compensating composite pulses ${ }^{17,18}$, can be calculated and experimentally validated as well (Fig. 1e). These protocols are commonly used in nuclear magnetic resonance and electron spin resonance in attempting to suppress static offsets in control parameters such as the frequency of the drive inducing spin rotations. Calculating the FFs for these protocols now reveals their sensitivity to time-dependent noise-an important characteristic for deployment in realistic quantum information settings ${ }^{19}$. We experimentally demonstrate a form of quantum system identification (Methods), effectively reconstructing the amplitude-noise filter functions, $F_{\Omega}(\omega)$, for two well-known compensating pulse sequences known by the shorthand designations SK1 and BB1 (Supplementary Methods). Again, calculations of $\mathcal{F}_{\chi}(\tau)$ match data well over the entire band in the weak-noise limit (Fig. 1f) with no free parameters.

Our choice of characterizing these compensating pulse sequences highlights an important issue in the prediction of ensembleaverage dynamics of controlled quantum systems. Ultimately, the underlying physical principles giving rise to the analytic form of $F(\omega)$ are based on the well-tested average Hamiltonian theory ${ }^{20}$ exploited in crafting these pulses. Despite this shared theoretical foundation, the calculation of spectral filtering properties is quite distinct from calculation of quasi-static error terms in a Magnus expansion, with important consequences for average quantum dynamics in realistic time-varying noise environments ${ }^{10}$.

Accordingly, our tests of the FF formalism reveal that compensating pulses designed to suppress errors to high order in a Magnus-expansion framework need not be efficient noise spectral filters (Supplementary Methods and ref. 19). Despite significant differences in their construction-the BB1 protocol is designed to provide higher-order cancellation of Magnus terms than SK1both of the selected composite pulses provide similar filtering of time-dependent noise, given by the filter order (slope of the FF in Fig. 1e). In the weak-noise limit frequency-domain characteristics 
a
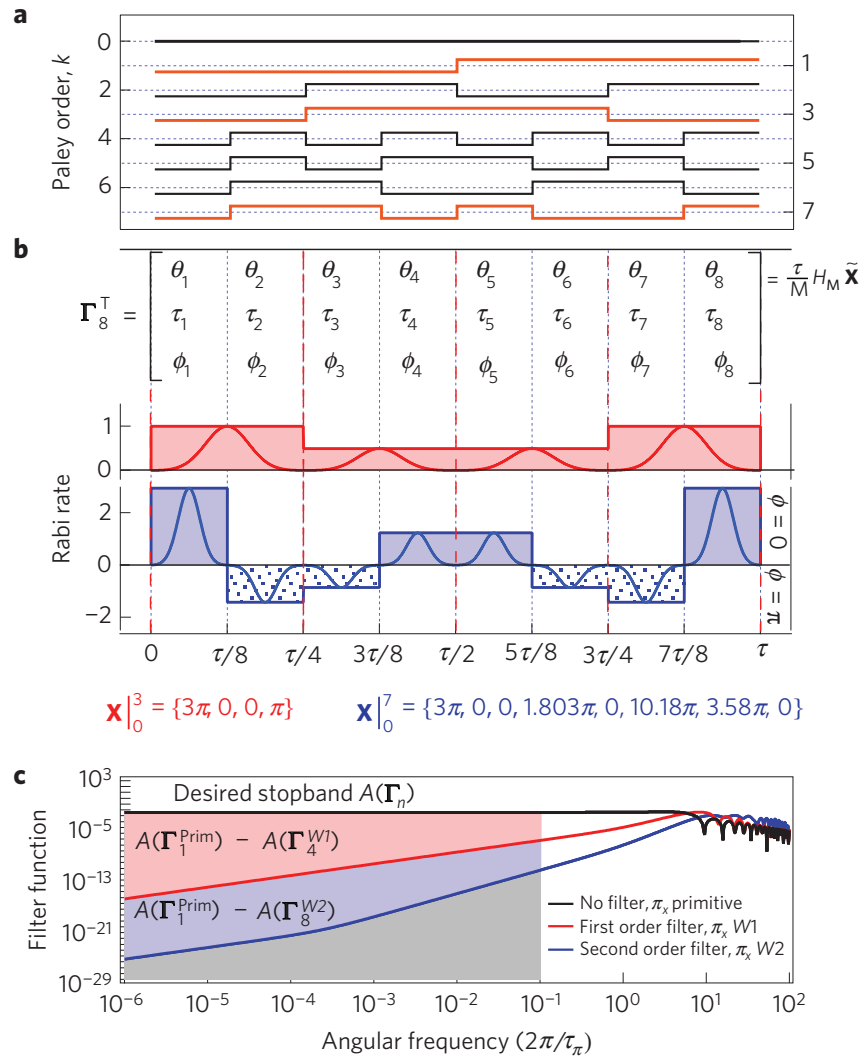

Figure 2 | Synthesis of high-pass amplitude-modulated filters from the Walsh functions. a, The first eight Walsh functions used in filter synthesis, $\left\{\mathrm{PAL}_{0}, \mathrm{PAL}_{7}\right\}$, with maximum-Hamming-weight-indexed functions highlighted in red. $\mathbf{b}$, Representative amplitude profiles for filter constructions found via numerical search over the Walsh basis with four (red, denoted W1) and eight (blue, denoted W2) time steps. Vertical axis represents $\Omega$, the Rabi rate per time step; negative values indicate $\pi$-phase shifts. Synthesis may be performed over square (flat-top) pulse segments or Gaussian-shaped pulse segments, with results differing only in the resulting Walsh coefficients. The matrix representing filter characteristics over eight segments is superimposed on the amplitude profiles (for $n=4$, neighbouring segments between red dashed lines are combined). The first row (the angles of rotation in each segment of the filter) is determined via Walsh synthesis, indicated by the vectors $\left.\mathbf{X}^{T}\right|_{0^{\prime}} ^{n}$ containing the spectral weights over $\mathrm{PAL}_{0} \rightarrow \mathrm{PAL}_{n}$. In the case of Gaussian pulse envelopes Walsh synthesis sets the first line, $\theta_{l}$. The symbol $\tilde{\mathbf{X}}$ indicates reordering for Hadamard synthesis, with listed coefficients appropriate for square pulse envelopes. c, The filter-transfer function for a primitive $\pi_{x}$ rotation and for synthesized noise filters. Performance improvement over the desired stopband of the filter captured in cost function $A\left(\Gamma_{4(8)}^{W 1\left(W_{2}\right)}\right)$ and its difference relative to that for the primitive operation, $A\left(\boldsymbol{\Gamma}_{1}^{\text {Prim }}\right)$. Filter $W 1$ gives improvement indicated by the red shading, with additional improvement in the cost function given by $W 2$ indicated by blue shading.

are captured accurately through the FF across frequencies ranging from quasi-static to rapidly fluctuating on the timescale of the pulse (Fig. 1f). Performance deviations between the pulses arise and the FF approximation breaks down as the noise strength is increased and higher-order terms in the Magnus series become important, but only at low frequencies (Fig. 1g). At frequencies fast relative to the control the FF again accurately predicts the relevant quantum dynamics even in the strong-noise limit. This is the first direct manifestation of the difference between studying quantum dynamics in terms of frequency-domain noise filtering and calculation of error contributions in a Magnus expansion as is appropriate in the quasi-static limit.
These simple but powerful validations of the predictive power of the generalized FF formalism now open the possibility of demonstrating the construction of noise filters with a specified spectral response, employing the filter-transfer functions as key analytic tools. Filters may take a wide variety of forms as needed by users-including high-pass filters for broadband noise suppression and band-stop filters useful for narrowband noise characterization (Fig. 1b).

In the discussion that follows, we focus on a common setting in which we aim to improve operational fidelity by reducing the influence of broadband non-Markovian noise on a target state transformation. Filters are realized as $n$-step sequences of time-domain control operations with tunable pulse amplitude and phase, similar in spirit to compensating composite pulses in NMR (refs 17-19), dynamically corrected gates (DCGs) in quantum information ${ }^{21,22}$, and open-loop modulated pulses in quantum control $^{23,24}$. However, recalling the difference between Magnus cancellation order and filtering order described above, in this setting we wish to synthesize a filter with arbitrary, user-defined spectral characteristics captured by a cost function, $A\left(\boldsymbol{\Gamma}_{n}\right)$, to be minimized for a filter represented by $\boldsymbol{\Gamma}_{n}\left(\theta_{l}, \tau_{l}, \phi_{l}\right)$ (Fig. 2b,c and Methods).

To provide efficient solutions to filter design we restrict our control space and focus on constructions synthesized using concepts from functional analysis in the basis set of Walsh functions-squarewave analogues of the sines and $\operatorname{cosines}^{4,25,26}$ (Fig. 2a). This approach provides significant benefits for our problem ${ }^{26}$, but is by no means the only basis set for composite filter construction ${ }^{27,28}$.

As an example we synthesize noise filters via weighted linear combinations of Walsh functions, $\mathrm{PAL}_{k}(x)$ denoted by the Paleyordered index, $k$. These filters are designed to suppress time-varying dephasing noise over a low-frequency stopband while implementing a bounded-strength driven rotation about the $x$ axis on the Bloch sphere (Supplementary Methods). In this case the Walshsynthesized waveform dictates an amplitude modulation pattern for the control field over discrete time segments. Importantly, Walsh filter synthesis is compatible with pulse segments possessing arbitrary pulse envelopes, including sequences of, for example, square (used here) or Gaussian pulse segments (Fig. 2b).

Analytic design rules provide simple insights into how one may craft effective modulation protocols, and a Nelder-Mead simplex optimization is used to find high-performing operations as defined by our cost function. Relative to an unfiltered primitive gate, the dephasing filter function, $F_{z}(\omega)$, for the simplest four-pulse construction $W 1$ shows increased steepness in the stopband (Fig. 2c, red), reducing $A\left(\boldsymbol{\Gamma}_{4}\right)$ (here the gate performs $\theta=\pi$ ). This measure of filter order may be further increased via construction $W 2$, in turn reducing the cost function for optimization (blue shaded area in Fig. 2c). Relating back to earlier demonstrations of filter order in compensating pulses, $W 2$ presents an interesting case of a highorder noise filter over the target band which provides only firstorder Magnus cancellation.

Filters $W 1$ and $W 2$ are representative, rather than unique solutions. In Fig. $3 \mathrm{~b}$ we show the calculated cost function, $A\left(\boldsymbol{\Gamma}_{4}\right)$, as a function of the Walsh coefficients used in constructing $W 1, X_{0}$ and $X_{3}$, giving the modulation profile indicated in Fig. 3a. Blue areas meet our minimized target, indicating useful filters, revealing a wide variety of possible constructions with favourable characteristics. Experimental tests of these protocols reveal that Walsh-modulated waveforms minimizing $A\left(\boldsymbol{\Gamma}_{4}\right)$ effectively suppress noise in the designated stopband for arbitrary rotation angles (Fig. $3 c-e$ ), and outperform standard pulses in the small-error limit germane to quantum information (Fig. 3f). See Methods.

Our focus has been on providing a validated framework for the vital task of predicting quantum dynamics in realistic environments and demonstrating the relevant physics through construction of noise spectral filters. The Walsh-modulated filters 

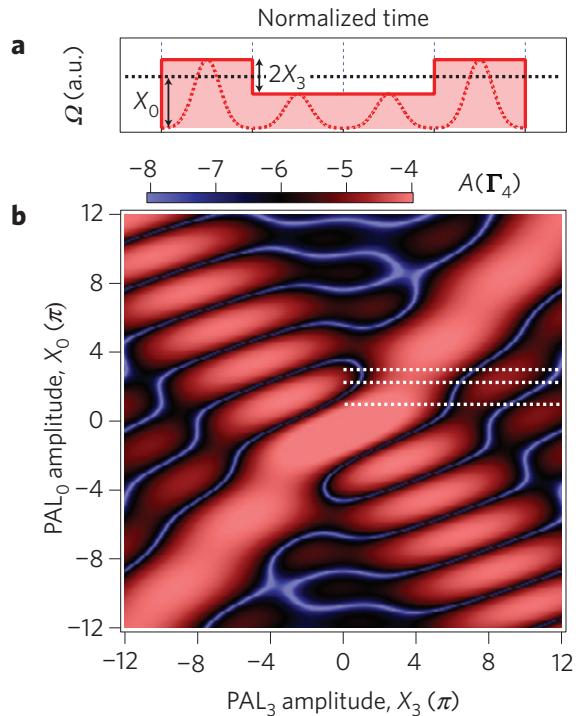

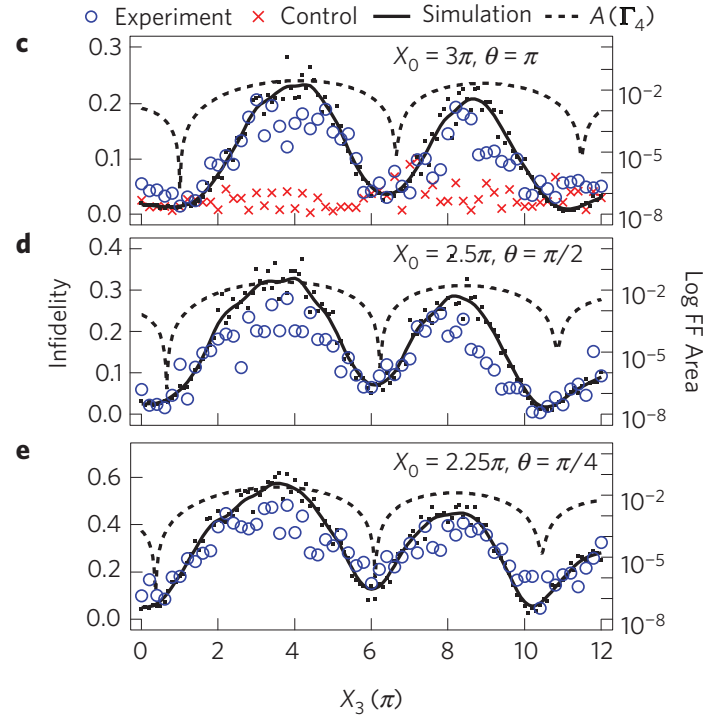

Figure 3 | Construction of the first-order Walsh amplitude-modulated dephasing-suppressing filter. a, Schematic representation of Walsh synthesis for a four-segment amplitude-modulated filter (WAMF). Walsh synthesis can be used to determine either the modulating envelope of square pulse segments, or the net area of discrete Gaussian pulses with differing amplitudes. b. Two-dimensional representation of the integral metric defining our target cost function, $A\left(\boldsymbol{\Gamma}_{4}\right)$ integrated over the stopband $\omega \in\left[10^{-9}, 10^{-1}\right] \tau^{-1}$. Areas in blue minimize $A\left(\boldsymbol{\Gamma}_{4}\right)$, representing effective filter constructions. The $X_{0}$ determines the net rotation enacted in a gate while $X_{3}$ determines the modulation depth, as represented in $\mathbf{a}$. White lines indicate possible constructions for filters implementing rotations of $\theta=\pi, \theta=\pi / 2$ and $\theta=\pi / 4$ from top to bottom. c-e, Experimental measurement of gate infidelity (left axis) for rotations constructed from various Walsh coefficients in the presence of engineered noise $\left(\omega_{c} / 2 \pi=20 \mathrm{~Hz}\right)$. Black dots and line represented calculated fidelity by Schroedinger equation integration (raw and smoothed respectively). All values of $X_{3}$ for a given $X_{0}$ implement the same net rotation, indicated by a control experiment with no noise. Total rotation time is scaled with $X_{3}$ to preserve a maximum Rabi rate. Black dashed line (right axis) corresponds to $A\left(\boldsymbol{\Gamma}_{4}\right)$ from $\mathbf{a}$. In experiments we always perform a net $\pi$ rotation $|0\rangle \rightarrow|1\rangle$ by sequentially performing identical copies of rotations for $\theta<\pi$. $\mathbf{f}$, Randomized benchmarking results (50 randomizations) demonstrating superior performance of a modulated gate in the small-error limit (infidelity $<0.5 \%$ per gate), see Supplementary Methods.

presented here-based on the achievable frequency-domain filter order-complement existing techniques rather than attempting to provide optimal-performance error-robust gates. Our results on high-pass noise filters, for instance, add to existing compensating pulse sequences designed for quasi-static noise, as well as gate constructions with interleaved dynamical decoupling that seek to periodically 'refocus' quantum evolution ${ }^{29-32}$.

Importantly, recent work has demonstrated that the filtertransfer function formalism is applicable to multi-qubit settings where dynamics may be considerably more complex than the single-qubit case ${ }^{33-35}$. In addition, ongoing efforts suggest there exists a path towards further extension of the generalized filtertransfer function and noise filtering formalisms to arbitrary control settings involving multiple qubits subject to general noise from nonMarkovian classical and/or quantum mechanical environments. We believe that with the validations provided here, this simple extensible framework with precise predictive power will provide a path for experimentalists to characterize and suppress the effects of noise in generic quantum coherent technologies, ultimately enabling a new generation of engineered quantum systems.

\section{Methods}

The fidelity of a control operation for a single qubit in the presence of a time-dependent environment is reduced as $\mathcal{F}_{\chi}(\tau)=1 / 2\left(1+\mathrm{e}^{-\chi(\tau)}\right)$, where $\chi(\tau)=1 / \pi \sum_{i} \int_{0}^{\infty} \mathrm{d} \omega S_{i}(\omega) F_{i}(\omega)$, and $\tau$ is the total duration of the operation. In this expression for fidelity, the integral considers contributions from independent noise processes through their frequency-domain power spectra $S_{i}(\omega), i \in\{z, \Omega\}$, capturing dephasing along $\hat{z}$ and amplitude noise co-rotating with a resonant drive field (Supplementary Methods). We employ here the so-called modified filter-transfer function, which subsumes a factor of $\omega^{-2}$ into the definition of $F_{i}(\omega)$. See refs 6,14 for details.

Experimental measurements involve state initialization in $|0\rangle$ followed by a control operation - or series of control operations-designed to drive qubit population from the dark state to the bright state, $|0\rangle \rightarrow|1\rangle$. For instance, tests of filters used for rotations $\theta<\pi$ are repeated sequentially such that the net rotation enacts $|0\rangle \rightarrow|1\rangle$ (Fig. 3d,e). The operational fidelity is measured as the probability that the qubit is in the bright state over an ensemble of measurements. Typical experimental uncertainties are limited by measurement fidelity $(\sim 98.5 \%)$ and quantum projection noise with maximum value comparable to the measurement infidelity for qubit states near the equatorial plane of the Bloch sphere. In general, a non-Markovian noise bath is engineered with specific properties of interest (see Supplementary Methods for full details). Additional measurement uncertainty of order $\sim 3-5 \%$ is added through finite sampling of the infinite ensemble of possible noise realizations. This is visible as fluctuations between neighbouring points in, for example, Fig. $3 \mathrm{c}-\mathrm{f}$.

Measurements in Fig. $1 \mathrm{c}$ are conducted for a simple $\pi_{x}$ enacted while varying the high-frequency cutoff, $\omega_{c}$, of a flat-top engineered non-Markovian dephasing bath (Fig. 1d). As the high-frequency cutoff of the noise is increased and fluctuations fast relative to the control $\left(\tau_{\pi}\right)$ are added to the noise power spectrum, $S_{z}(\omega)$, errors accumulate, reducing the measured fidelity. For $\omega_{c} / 2 \pi=1$ the highest frequency contribution to $S_{z}(\omega)$ undergoes a complete cycle of oscillation over $\tau_{\pi}$, indicating that the noise is time-dependent on the scale of a single experiment even for $\omega_{c} / 2 \pi \ll 1$. We calculate $\mathcal{F}_{\chi}(\tau)$ using the form of the noise and the analytic FF for a driven primitive gate under dephasing ${ }^{10}$, finding good agreement with experimental measurements using no free parameters.

Measurements in Fig. 1f,g employ a narrowband 'delta-function' noise power spectrum swept as an experimental variable, $\omega_{t}$. Injected noise takes the form of fixed-frequency amplitude modulation of the near-resonant driving field during application of a control pulse, with strength (modulation depth) parameterized in terms of $\Omega$, the Rabi rate of the drive. The form of $\mathcal{F}_{x}(\tau)$ demonstrates that the calculated fidelity involves an exponentiated value of the FF at frequency $\omega_{t}$, meaning that fidelity measurements effectively reconstruct the filter functions. Key features in the data, such as performance-crossover frequencies between primitive and compensating gates and deep notches in the filter at high frequency, are quantitatively reproduced in experimental measurements.

Filter construction presented in Figs 2 and 3 is parameterized as a function of controllable properties of a near-resonant carrier frequency enacting driven operations. An arbitrary $n$-segment filter is represented over successive timesteps through the matrix quantity $\boldsymbol{\Gamma}_{n}\left(\theta_{l}, \tau_{l}, \phi_{l}\right)$ (Fig. 2b); in each segment of duration $\tau_{l}$ 
we perform a driven operation generating a rotation through an angle $\theta_{l}=\int_{t_{l-1}}^{t_{l}} \Omega_{l}(t) \mathrm{d} t$ about the axis $\mathbf{r}_{l}=\left(\cos \left(\phi_{l}\right), \sin \left(\phi_{l}\right), 0\right)$, with $\Omega_{l}(t)$ the Rabi rate over the $l$ th pulse segment.

The value of $n$ is chosen to be a power of two, compatible with synthesis over discrete-time Walsh functions. The Walsh functions are piecewise-constant over segments which are all integer multiples of base period $\tau_{l}$. This approach brings benefits for the current setting ${ }^{26}$; for instance, their piecewise-constant construction builds intrinsic compatibility with discrete clocking and classical digital logic, while the well-characterized mathematical properties of the Walsh functions provide a basis for establishing simple analytic filter-design rules, and flexibility in realizing a wide variety of filter forms.

For the filters $W 1$ and $W 2$ presented in the main text, Walsh-synthesis design rules dictate that we implement our filtered rotation by $\theta_{x}$ over a minimum of four discrete steps, permitting synthesis over $\mathrm{PAL}_{0}$ to $\mathrm{PAL}_{3}$. Within this small set, the coefficient of $\mathrm{PAL}_{0}$, denoted $X_{0}$, sets the total rotation angle $\theta \bmod 2 \pi$ for the modulated driven evolution, and only non-zero $X_{3}$ preserves symmetry. We experimentally test the performance of four-segment amplitude-modulated filters by scanning over $X_{3}$ for fixed $X_{0}$ (denoted by white dotted lines in Fig. 3b). Values of $X_{3}$ minimizing $A\left(\boldsymbol{\Gamma}_{4}\right)$ (dips in the dashed trace, right axis) also minimize the experimentally measured infidelity in the presence of engineered low-frequency noise (open circles, left axis). This behaviour is observed for various target rotation angles of interest (Fig. $3 \mathrm{c}-\mathrm{e}$ ), with predicted shifts in the optimal values of $X_{3}$ with changes in $X_{0}$ borne out through experiment. Filter $W 2$ is constructed over $\mathrm{PAL}_{0}$ to $\mathrm{PAL}_{7}$, and has twice as many timesteps as $W 1$. Interestingly, $W 1$ is a special case of an analytically constructed dynamically corrected NOT gate (a $\pi$-rotation $)^{21}$. For details of the Walsh functions, Walsh synthesis and Walsh-basis analytic design rules see Supplementary Methods.

Received 1 April 2014; accepted 2 September 2014; published online 19 October 2014

\section{References}

1. Smith, A. et al. Quantum control in the ${ }^{6} \mathrm{Cs}_{1 / 2}$ ground manifold using radio-frequency and microwave magnetic fields. Phys. Rev. Lett. 111, 170502 (2013).

2. Dolde, F. et al. Electric-field sensing using single diamond spins. Nature Phys. 7, 459-463 (2011).

3. Kucsko, G. et al. Nanometre-scale thermometry in a living cell. Nature $\mathbf{5 0 0}$, 54-58 (2013)

4. Cooper, A., Magesan, E., Yum, H. \& Cappellaro, P. Time-resolved magnetic sensing with electronic spins in diamond. Nature Commun. 5, 3141 (2014),

5. Álvarez, G. A. \& Suter, D. Measuring the spectrum of colored noise by dynamical decoupling. Phys. Rev. Lett. 107, 230501 (2011).

6. Bylander, J. et al. Noise spectroscopy through dynamical decoupling with a superconducting flux qubit. Nature Phys. 7, 565-570 (2011).

7. Clark, J. W., Lucarelli, D. G. \& Tarn, T. J. Control of quantum systems. Int. J. Mod. Phys. B 17, 5397-5411 (2003).

8. Bouten, L., Handel, R. V. \& James, M. R. An introduction to quantum filtering. SIAM J. Control Optim. 46, 2199-2241 (2007).

9. Green, T. J., Uys, H. \& Biercuk, M. J. High-order noise filtering in nontrivial quantum logic gates. Phys. Rev. Lett. 109, 020501 (2012).

10. Green, T. J., Sastrawan, J., Uys, H. \& Biercuk, M. J. Arbitrary quantum control of qubits in the presence of universal noise. New J. Phys. 15, 095004 (2013).

11. Kofman, A. \& Kurizki, G. Universal dynamical control of quantum mechanical decay: Modulation of the coupling to the continuum. Phys. Rev. Lett. 87, 270405 (2001).

12. Uhrig, G. Keeping a quantum bit alive by optimized pi-pulse sequences. Phys. Rev. Lett. 98, 100504 (2007).

13. Cywinski, L., Lutchyn, R. M., Nave, C. P. \& Sarma, S. D. How to enhance dephasing time in superconducting qubits. Phys. Rev. B 77, 174509 (2008).

14. Biercuk, M. J., Doherty, A. C. \& Uys, H. Dynamical decoupling sequence construction as a filter-design problem. J. Phys. B 44, 154002 (2011).

15. Biercuk, M. J. et al. Optimized dynamical decoupling in a model quantum memory. Nature 458, 996-1000 (2009).

16. Soare, A. et al. Experimental bath engineering for quantitative studies of quantum control. Phys. Rev. A 89, 042329 (2014).
17. Vandersypen, L. M. K. \& Chuang, I. NMR techniques for quantum control and computation. Rev. Mod. Phys. 76, 1037-1069 (2004).

18. Merrill, J. T. \& Brown, K. R. in Quantum Information and Computation for Chemistry: Advances in Chemical Physics Vol. 154 (ed. Kais, S.) Ch. 10, 241-294 (John Wiley, 2014).

19. Kabytayev, C. et al. Robustness of composite pulses to time-dependent control noise. Phys. Rev. A 90, 012316 (2014)

20. Haeberlen, U. \& Waugh, J. S. Coherent averaging effects in magnetic resonance. Phys. Rev. 175, 453-467 (1968).

21. Khodjasteh, K. \& Viola, L. Dynamically error-corrected gates for universal quantum computation. Phys. Rev. Lett. 102, 80501 (2009).

22. Wang, X. et al. Composite pulses for robust universal control of singlet-triplet qubits. Nature Commun. 3, 997 (2012).

23. Fauseweh, B., Pasini, S. \& Uhrig, G. Frequency-modulated pulses for quantum bits coupled to time-dependent baths. Phys. Rev. A 85, 022310 (2012).

24. Ticozzi, F., Nishio, K. \& Altafini, C. Stabilization of stochastic quantum dynamics via open and closed loop control. IEEE Trans. Auto. Control 58, 74-85 (2013).

25. Beauchamp, K. G. Walsh Functions and their Applications (Academic, 1975).

26. Hayes, D., Khodjasteh, K., Viola, L. \& Biercuk, M. J. Reducing sequencing complexity in dynamical quantum error suppression by Walsh modulation. Phys. Rev. A 84, 062323 (2011).

27. Owrutsky, P. \& Khaneja, N. Control of inhomogeneous ensembles on the Bloch sphere. Phys. Rev. A 86, 022315 (2012).

28. Jones, N. C., Ladd, T. D. \& Fong, B. H. Dynamical decoupling of a qubit with always-on control fields. New J. Phys. 14, 093045 (2012).

29. Barthel, C., Medford, J., Marcus, C. M., Hanson, M. P. \& Gossard, A. C. Interlaced dynamical decoupling and coherent operation of a singlet-triplet qubit. Phys. Rev. Lett. 105, 266808 (2010).

30. Liu, G-Q., Po, H. C., Du, J., Liu, R. B. \& Pan, X. Y. Noise-resilient quantum evolution steered by dynamical decoupling. Nature Commun. 4, 2254 (2013)

31. Souza, A. M., Álvarez, G. A. \& Suter, D. Experimental protection of quantum gates against decoherence and control errors. Phys. Rev. A 86, 050301 (2012).

32. van der Sar, T. et al. Decoherence-protected quantum gates for a hybrid solid-state spin register. Nature 484, 82-86 (2012).

33. Su, Z-K. \& Jiang, S-J. Filter-design perspective applied to dynamical decoupling of a multi-qubit system. J. Phys. B 45, 025502 (2012).

34. Silva, G. P. \& Viola, L. A general transfer-function approach to noise filtering in open-loop quantum control. Preprint at http://arXiv.org/abs/1408.3836 (2014).

35. Green, T. J. \& Biercuk, M. J. Phase-modulated decoupling and error suppression in qubit-oscillator systems. Preprint at http://arXiv.org/abs/1408.2749 (2014).

\section{Acknowledgements}

We thank P. Fisk, M. Lawn, M. Wouters and B. Warrington for technical assistance and K. Brown, J. T. Merrill and L. Viola for useful discussions. This work partially supported by the US Army Research Office under Contract Number W911NF-11-1-0068, the Australian Research Council Centre of Excellence for Engineered Quantum Systems CE110001013, the Office of the Director of National Intelligence (ODNI), Intelligence Advanced Research Projects Activity (IARPA), through the Army Research Office, and the Lockheed Martin Corporation. X.Z. acknowledges useful discussions with G. L. Long and support from the National Natural Science Foundation of China (Grants No. 11175094 and No. 91221205) and the National Basic Research Program of China (No. 2011CB9216002).

\section{Author contributions}

A.S., H.B., D.H. and M.J.B. conceived and performed the experiments, built experimental apparatus, contributed to data analysis and wrote the manuscript. T.J.G. conceived the relevant theoretical constructs. J.S., M.C.J. and X.Z. assisted with development of the experimental system and data collection. J.J.M. assisted with data collection.

\section{Additional information}

Supplementary information is available in the online version of the paper. Reprints and permissions information is available online at www.nature.com/reprints. Correspondence and requests for materials should be addressed to M.J.B.

\section{Competing financial interests}

The authors declare no competing financial interests. 